\title{
Die neue Verfassung der Republik Südafrika - von "Westminster" nach "Southminster"
}

\author{
Von Klaus Frhr. von der Ropp
}

\section{I.}

Der langjährige Beobachter der politischen Szene Südafrikas bemerkt seit etwa zwei oder drei Jahren, daß ihm das weiße Südafrika jetzt wieder mit jener Selbstsicherheit gegenübertritt, die sein Auftreten so sehr lange gekennzeichnet hatte, die ihm dann aber Mitte der 70er Jahre abhanden gekommen war. Seinerzeit hatten die Ablösung der portugiesischen Kolonialverwaltung in Mozambique und Angola durch "marxistische" Regime, die Verschlechterung der militärischen Lage der weißen Minderheitsregierung unter Ian Smith im damaligen Rhodesien, die über 18 Monate währende Rebellion des schwarzen Südafrikas ("Soweto", "Kreuzzug der Kinder") und vielleicht noch mehr die lautstark und in einer zur Eile gemahnenden Sprache vorgebrachte Forderung führender Westmächte nach einer Machtumkehr in Windhoek und in Pretoria das weiße Südafrika zutief st verunsichert. Viele der herrschenden Weißafrikaner dachten damals über Rückfallpositionen, über Kompromißformeln nach, da auch sie den status quo für auf Dauer unhaltbar hielten. Ein deutsch-südafrikanisch-niederländisches Forschungsprojekt des Arnold-Bergstraesser-Instituts (Freiburg) ${ }^{1}$ zur weißafrikanischen Wandlungsbereitschaft (und den schwarzafrikanischen Wandlungserwartungen) im Sozialen, Wirtschaftlichen und Politischen zeigte ein bis dahin unbekanntes Maß an Bereitschaft zum Verzicht auf bestehende Privilegien an.

Die Unsicherheit jener Jahre ist heute weitgehend gewichen. Dafür zeichnen eine ganze Reihe von Faktoren verantwortlich. Bis zum Ende der 70er Jahre war es, ohne das in Südafrika übliche $M a ß$ an Repression sonderlich zu überziehen, Pretoria gelungen, die schwarze Rebellion, der sich auch gemischtrassige und indienstämmige Südafrikaner angeschlossen hatten, zu ersticken. Entsprechend vermochte Pretoria das zuvor verlorengegangene Vertrauen westlicher Wirtschaftskreise zurückzugewinnen. Südafrika mit seinen immensen Vorkommen an häufig seltenen und wertvollen Rohstoffen wurde wieder zum bevorzugten Handels- und Investitionspartner. Auch bewies Südafrika immer wieder seine Fähigkeit, Guerilleros der wichtigsten südafrikanischen Befreiungsbewegung, des African National Congress (ANC), bereits in ihren Ausgangsstellungen in Nachbarstaaten anzugreifen und auszuschalten. Entsprechend konnte die Zahl der in Südafrika begangenen Anschläge auf Einrichtungen der Infrastruktur und solche des

1 Theo Hanf, Heribert Weiland und Gerda Vierdag (unter Mitarbeit von Lawrence Schlemmer u. a.) "Südafrika: Friedlicher Wandel ..., 1978. 
Militärs, der Polizei usw., so spektakulär sie im Einzelfall auch waren, auf einem durchaus erträglichen Niveau gehalten werden. Wichtiger noch war, daß die Westmächte auf Ubergriffe der südafrikanischen Streitkräfte auf Ziele in Angola, Mozambique und Lesotho sowie auf Maßnahmen der wirtschaftlichen Destabilisierung in anderen Anrainern $^{2}$ in jüngerer Zeit zunehmend milder, ja fast resignierend reagierten. Die noch dazu seltenen, von keinem Veto eines westlichen Staates verhinderten Verurteilungen Südafrikas durch den UN Sicherheitsrat zeigten keine Wirkung, da sie ohne Folgen blieben. In der Rolle einigermaßen hilfloser Akteure werden die Westmächte heute Zeuge einer südafrikanischen Außen- und Sicherheitspolitik, die Anrainer (Swaziland, Lesotho, Mozambique und Angola u. a.) in absehbarer Zeit möglicherweise zwingen wird, mit Pretoria sog. Antisubversionsabkommen abzuschließen. D. h., daß sich beide Seiten verpflichten, die Regierung des jeweiligen Vertragspartners nicht mehr zu destabilisieren. An der Südspitze Afrikas regiert derzeit nur noch die regionale Supermacht Südafrika.

\section{Das Versagen westlicher Politik und seine Folgen für einen innersüdafrikanischen Ausgleich}

Viel relevanter als alles Vorstehende für die Zukunft Südafrikas war aber, daß die Republik Südafrika, genauer: das Volk der nur 5 Mio. weißen Afrikaner über alle Versuche der westlichen Fünf triumphierte, sie zum Abzug aus SWA/Namibia und zur Aufgabe der Politik der Apartheid zu zwingen.

Die im März 1977 begonnene SWA/Namibia-Initiative der seinerzeitigen westlichen Sicherheitsratsmitglieder USA, Großbritannien, Bundesrepublik Deutschland, Frankreich und Kanada, der nach den Vorstellungen ihrer Väter (Jimmy Carter, Andrew Young und Hans-Dietrich Genscher) eine analoge Südafrika-Initiative folgen sollte, ist auf der ganzen Linie kläglich gescheitert. In den zurückliegenden Jahren sind die "Fünf" vor den Nachkommen jener 75000 afrikaansen Terroristen, die das britische Weltreich um die Jahrhundertwende zur Entsendung von nahezu einer halben Million Soldaten in den Süden Afrikas gezwungen hatten, in die Rolle ohnmächtiger Zuschauer gedrängt worden.

Mit der SWA/Namibia-Initiative scheiterten naturgemäß auch alle Vorstellungen der "Fünf", in Südafrika selbst fundamentale Änderungen zu erwirken. Von nicht zu überschätzender Bedeutung ist, daß die "Fünf" nicht, wie oft behauptet, erst in der Amtszeit Ronald Reagans scheiterten. Sie unterlagen Pretoria bereits in der Amtszeit von Jimmy Carter, der, anders als sein Nachfolger, an den Problemen Südafrikas ein sehr großes, auch persönliches Interesse hatte. Das von Reagans Assistant Secretary of State for

2 Simon Jenkins "Destabilisation in Southern Africa/Potgieter counter-attacks" in The Economist vom 16. Juli 1983, S. 15-28; Deon Geldenhuys "The Destabilisation Controversy: An Analysis of a High-Risk Foreign Policy Option for South Africa" in Politikon (Pretoria), Dezember 1982, S. 16-31. Aus dem deutschsprachigen Raum s. vor allem den hervorragenden Beitrag von Gerald Braun, "Die regionale Wirtschaftsstrategie der Republik Südafrika: Von der wirtschaftlichen Kooperation zur ökonomischen Destabilisierung", hrsg. vom ABI, Freiburg 1984. 
African Affairs, Chester Crocker, und dessen Stellvertreter Frank Wisner ins Spiel ge| brachte "Cuban linkage" hat, aus heutiger Perspektive, die Entlassung SWAs/Namibias in die Unabhängigkeit wohl weiter erschwert. Bereits zuvor aber hatten die "Fünf", und unter ihnen vor allem Washington, Bonn und das allerdings nur eine Nebenrolle spielende Ottawa, irreparable Fehler gemacht.

Hier ist nicht der Raum, den Gang der seinerzeitigen Verhandlungen in allen Einzelheiten $^{3}$ nachzuzeichnen. Erwähnt sei jedoch, daß die Politik der "Fünf" an einer schwer nachvollziehbaren Konzeptionslosigkeit litt. So scheint bei etlichen der hier involvierten westlichen Regierungen jede Vorstellung von dem Widerstand gefehlt zu haben, den Pretoria dem Westen entgegenzusetzen entschlossen war. ${ }^{4}$ Dies kam etwa darin zum Ausdruck, daß führende Repräsentanten Washingtons, Bonns und Ottawas (nicht jedoch der mit afrikanischen Fragen so detailliert vertrauten Regierungen in Paris und London) nicht müde wurden, selbst vor dem UN Sicherheitsrat zu äußern, die Lösung ("oneman-one-vote"), die man für (die europäisch-südafrikanische Siedlerkolonie) SWA/Namibia gefunden habe, habe für (den Mehrvölkerstaat) Südafrika Modellcharakter. Dem letzteren widersprachen in Südafrika nicht nur die in der ausgeprägt konservativen (regierenden) Nasionale Party van Suid-Afrika (NP) oder der erst später gegründeten reaktionären Konserwatieve Party (KP) organisierten Weißen sondern auch die eher linksliberalen Führungszirkel der liberalen weißen Oppositionspartei, der Progressive Federal Party (PFP). So sehr unterschiedlich ihre Vorstellungen von einer künftigen politischen Ordnung für Südafrika auch sind, so stimmen sie doch alle darin überein, daß ihre kulturelle, soziale, wirtschaftliche und politische Identität als einer in mehr als drei Jahrhunderten gewachsenen weißafrikanischen Nation nicht zur Disposition stehe. Die Forderungen aus Bonn, Washington und Ottawa besagten aber letztlich nichts anderes, als daß das der Ersten Welt zugehörige weiße Südafrika (d. s. ca. 17 v. H. von einer Gesamtbevölkerung von ca. $30 \mathrm{Mio}$ ) sich bedingungslos der übergroßen schwarzen Mehrheit (ca. 70 v. H. der Gesamtbevölkerung) unterzuordnen habe, d. h. auf seine Jdentität verzichten müsse. In allen westlichen Hauptstädten hätte bekannt sein muissen, daß das weiße Südafrika militärisch und ökonomisch einfach zu mächtig war, um sich selbst aufzugeben. M. a. W., man hätte schon sehr frühzeitig erkennen müssen, daß man auf dem eingeschlagenen Weg scheitern werde. Das aber war, aus welchen Gründen auch immer, nicht der Fall.

Nur wenige jener Offiziellen, die von Anfang 1977 bis Anfang 1981 in Washington, Bonn und Ottawa für die Formulierung der Afrikapolitik verantwortlich zeichneten, bewiesen das Einfühlungsvermögen Egon Bahrs. In einem Interview ${ }^{5}$ wies er darauf hin,

3 Dazu Klaus Frhr. von der Ropp "Konfliktherde im südlichen Afrika - Rhodesien, Namibia, Südafrika" in Wolfgang Wagner u. a. (Hrsg.) "Die Internationale Politik 1977/78", 1982, S. 269-285; ders. "Südafrika und Namibia: Das Scheitern der Friedensinitiativen" in Wolfgang Wagner u. a. (Hrsg.), "Die Internationale Politik 1979/80", 1983, S. 338-357; ders. "Südafrika - Chance und Herausforderung für die Liberalen" in liberal (Bonn) 1983. November, S. 855-867.

4 S. Klaus Frhr. von der "Frieden oder Krieg im südlichen Afrika" in Außenpolitik 4/1977, S. 437-454.

5 In Deutsches Allgemeines Sonntagsblatt vom 10. Juli 1977, S. 8. 
daß es zwischen den Problemen SWAs/Namibias und Rhodesiens/Zimbabwes einerseits und denen Südafrikas andererseits fundamentale Unterschiede gebe. Anders als für die beiden Siedlerkolonien komme für Südafrika eine staatliche Ordnung des "one-manone-vote" nicht in Betracht. Er argumentierte weiter ". . . und dann brauchen wir natürlich eine Regelung, die das Nebeneinander von Schwarz und Weiß mit der Sicherung der Rechte beider Rassen verbindet . . . also braucht man ein bisher unbekanntes Modell des gleichberechtigten Zusammenlebens mit besonderem Schutz für Minderheiten." Der spätere Parlamentarische Staatssekretär im Bundesministerium für Wirtschaftliche Zusammenarbeit, Volkmar Köhler, hat diese Uberlegungen später in einem Vortrag in Johannesburg aufgegriffen und um den alles entscheidenden Punkt ergänzt "Nur derjenige vermag dem schwarzen Südafrika die Freiheit zu bringen, der auch das Existenzrecht der dort lebenden weißafrikanischen Nation absichert . . . Wer das nicht sieht, der bewirkt wenig mehr, als daß er Südafrika an einen Abgrund schrecklicher Gewalt führt . . ." ${ }^{6}$ Zwei Jahre zuvor hatte übrigens die FDP in ihrem hier gemeinsam mit dem PFP-Vorsitzenden formulierten Programm für die Bundestagswahl von 1980 festgestellt, daß die durch Verhandlungen zu erzielende neue Verfassung Südafrikas das Selbstbestimmungsrecht der Mehrheit und die Absicherung des Existenzrechts der Minderheiten als voneinander abhängige Elemente enthalten müsse. ${ }^{7}$ Es war für den Erfolg nicht nur der westdeutschen Politik fatal, daß solche Uberlegungen nie in die offiziellen Reden der seinerzeit Verantwortlichen aufgenommen wurden.

Die Regierung in London und Paris suchten in der selben Richtung wie Egon Bahr, vermochten jedoch nicht zu verhindern, daß die hier federführenden Regierungen in Washington und Bonn bei ihrem einmal eingeschlagenen Kurs blieben.

Die Regierung in Pretoria erkannte so schon sehr frühzeitig, daß sie mit einem für sie innenpolitisch sehr kostspieligen Rückzug aus SWA/Namibia für ihr so problematisches Verhältnis zur westlichen Welt nichts würde gewinnen können. Also galt es, die totgeborene SWA/Namibia-Initiative endgültig zum Scheitern zu bringen. Das gelang auch. Denn trotz eines enormen Aufwandes an außerordentlich intensiver Reise- und Konferenzdiplomatie hatten die "Fünf" der letztlich starren und doch geschickten Diplomatie Südafrikas nichts Gleichwertiges entgegenzusetzen. Ein weiterer Höhepunkt westlichen Versagens war die Konferenz, zu der sich im Oktober 1978 die Außenminister der USA, Großbritanniens, Kanadas, der Bundesrepublik Deutschland, Südafrikas sowie der Staatssekretär im französischen Außenministerium in Pretoria versammelten. Damals machte die Regierung von P. W. Botha, obwohl von den Vertretern der Westmächte beschworen, den in Res. 435 des Sicherheitsrates niedergelegten SWA/Namibia-Plan zu akzeptieren, ihren Gesprächspartnern klar, daß sie unter völliger Mißachtung eben dieses Planes ihren eigenen, "demokratischen" Weg in Winhoek gehen werde. Entgegen al-

6 In International Affairs Bulletin (Braamfontein/SA) "Europe's Role in Africa: A German View" 3/1982, S. 35-45.

7 Dazu Klaus Frhr. von der Ropp "Der Süden Afrikas im Zeichen des Umbruchs" in liberal 1980. Dezember, S. 940-948 (941). 
lerlei nicht nur offiziösen Drohungen aus früherer Zeit sahen sich die "Fünf " jetzt außerstande, Pretoria durch die Anwendung von Zwangsmitteln, darunter wirtschaftlichen Sanktionen, zum Einlenken zu zwingen. Die britische und die westdeutsche Volkswirtschaft wären in ihrer Abhängigkeit von südafrikanischen Rohstofflieferungen den Gegensanktionen Pretorias nicht gewachsen gewesen. Ubrigens wurden die Anfang 1979 bekanntgewordenen Pläne der Bundesregierung, für die hier besonders interessierenden Rohstoffe (Chrom, Mangan, Vandadium, Kobalt und bestimmte Asbestsorten) entsprechende Vorratslager anzulegen, dann schon im Herbst 1980 wegen angeblich fehlender Haushaltsmittel aufgegeben. Die Bundesrepublik blieb also, ähnlich Großbritannien, ein "captive ally" Südafrikas.

Die westliche Politik der späten 70er Jahre hat deutlich in der ohnehin zutiefst gespaltenen südafrikanischen Gesellschaft zu einer noch größeren Polarisierung geführt. Denn das schwarze Südafrika, ob nun moderaten oder militanten Gruppen zugehörig, wurde durch die siegesgewissen Reden auch sehr hoher westlicher Verantwortlicher in seinem Glauben an die Zwangsläufigkeit des eigenen Sieges bestätigt. Niemand unter den Schwarzen vermochte sich vorzustellen, daß den vielen offiziellen Reden und sonstigen Äußerungen des Westen keine Taten folgen würden. In demselben Maß wie Erwartungen der Schwarzen geweckt wurden, wurde die Angst der Weißen bis hin zur nackten Existenzangst geschürt. Selbst solche Weißen, die sonst ganz drastischen Reformen das Wort redeten, dachten jetzt vornehmlich über verbesserte Techniken bei der Bekämpfung von Guerilleros nach.

Ab Anfang 1981 betrieb Washington dann eine sehr viel kenntnisreichere, sehr viel nachdenklichere Politik im südlichen Afrika. Wohl auch in Erkenntnis der Erfolglosigkeit der eigenen bisherigen Politik paßte sich ihr die Bonner Politik, wenn auch nur zögernd, an. Allerdings blieb auch diese von dem so sehr fähigen Chester Crocker maßgeblich geprägte Politik bislang erfolglos. ${ }^{8}$ In den Jahren zuvor hatten nämlich die westlichen "Fünf" bei allen afrikanischen Beteiligten an Glaubwürdigkeit verloren. Weder die Parteien der schwarzen Namibianer und schwarzen Südafrikaner, noch die afrikanischen Frontstaaten, noch die weißen Afrikaner in SWA/Namibia und Südafrika hatten Grund, der Politik der "Fünf" Vertrauen zu schenken. Möglicherweise werden künftige Historiker urteilen, daß unter dem Scherbenhaufen westlicher Politik der 70er Jahre die letzte Chance begraben wurde, die Konflikte in und um SWA/Namibia und Südafrika ohne ein Maximum an beidseitiger militärischer Gewaltanwendung zu lösen.

\section{Entstehungsgeschichte und Strukturen der neuen Verfassung}

Die Vorstellungen Chester Crockers, Volkmar Köhlers und Egon Bahrs zielen darauf $a b$, daß der Westen darauf hinwirken möge, daß sich die legitimen Repräsentanten der

8 S. etwa Chester Crocker "South Africa: Strategy for Change" in Foreign Affairs (New York), 2/1980-81, S. 323-35i: ders. "A U.S. Policy for the 80s", SA Institute of International Affairs, Johannesburg, 1981. 
weißen, braunen und schwarzen Südafrikaner, ob derzeit nun frei, inhaftiert oder im Exil lebend, bei einer Nationalen Konvention über eine neue politische, wirtschaftliche und soziale Ordnung für Südafrika einigen sollten. Die Einberufung einer solchen Verfassungsgebenden Versammlung ist übrigens die zentrale Forderung des politischen Programms der PFP. Dieselbe Forderung wird von allem moderaten wie auch den militanten Gruppierungen der gemischtrassigen, indienstämmigen und schwarzen Südafrikaner erhoben.

Nachdem sich der Druck des Westens als eine stumpfe Waffe erwiesen hat, sieht man in Pretoria erst recht keinen Grumd mehr dafür, sich mit dem ANC und den anderen Parteien der Schwarzen auf einen Dialog über die Zukunft des Landes einzulassen. Denn ein solcher Dialog wäre für Pretoria voller unwägbarer Risiken. Zumindest aber müßte die weiße Seite, um ihn erfolgreich zu gestalten, unvorstellbar große Opfer erbringen. So zieht sie es in Anknüpfung an die eigene Geschichte und auch an das Vorgehens Israels im Nahen Osten vor, mit eiserner Faust zu reagieren. Entsprechend ist auch die jetzt vom Kapstädter Parlament ratifizierte Verfassung nicht mehr als ein nicht ungeschickt verschleiertes Diktat der Weißen an die Braunen. In Südafrika regiert also unverändert "baasskap", und daran wird sich auf absehbare Zeit kaum etwas ändern.

\section{1) Einige Anmerkungen zu der bisherigen Ordnung}

"Aparheid" gibt es in Südafrika weit länger als seit 1948, dem Jahr, da in freien Wahlen unter den weißen Südafrikanern die seinerzeit rein afrikaanse Nasionale Party van SuidAfrika (NP) im Parlament zu Kapstadt erstmals eine Mehrheit gewann. In dem an "Westminister" orientierten politischen System der Weißen hat sie seither alle Wahlen gewonnen und regiert auch heute noch unangefochten. Inzwischen gibt ihr nämlich auch ein großer Teil der englischsprachigen Weißen seine Stimme. Gefahr könnte ihr eines Tages von Seiten der KP drohen, sollte diese irgendwann mit der noch extremistischeren Herstigte Nasionale Party (HNP) zusammengehen. Die KP und noch stärker die HNP progagieren die Rückkehr zur rigiden Apartheid Verwoerdscher Prägung. In ihren Augen ist die NP unter P. W. Botha auf dem besten Weg, die Interessen der Weißen, insbesondere die des Afrikanerdoms, zu verspielen.

Die Politik der Apartheid zielt bekanntlich darauf ab, die den Schwarzen verbliebenen Siedlungsgebiete ("Black National States", "Bantustans", "Heimatländer"), die in Wahrheit nicht mehr als bloße Reservate sind, in die staatliche Unabhängigkeit zu entlassen. Diese Reservate befinden sich im Norden und Osten des Landes und haben die geographische Gestalt eines Flickenteppichs. Alle Reservate sind übervölkert. Ihre Bevölkerung wächst, abgesehen von dem überall im schwarzen Afrika anzutreffenden starken natürlichen Bevölkerungswachstum, auch dadurch unaufhörlich, daß die Regierungsbehörden stetig "unproduktive" oder "nicht mehr produktive" Schwarze in die Reservate abschieben. Hier sind in den zurückliegenden Jahren Millionen von Menschen zwangsweise umgesiedelt worden. Zusammen machen die Reservate knapp 13 v. H. des 
südafrikanischen Territoriums aus und erwirtschaften etwa $1 \mathrm{v} . \mathrm{H}$. des südafrikanischen Bruttosozialprodukts.

Vier dieser Armenhäuser (Transkei, Boputhatswana, Venda und Ciskei) wurden in den zurückliegenden Jahren in die (Schein-)Unabhängigkeit entlassen. Ein fünftes Reservat, gleichfalls von Quislingen regiert, KwaNdebele, soll ihnen 1984 folgen. Pretoria läßt nichts unversucht, auch die übrigen Reservate (Qwa-Qwa, Gazankulu, Lebowa, Kangwane sowie das von dem hervorragenden M. Gatsha Buthelezi geführte KwaZulu) in die "Unabhängigkeit" zu drängen. Mit der Entlassung in die "Unabhängigkeit" verlieren alle Angehörigen der jeweiligen Stämme gleichgültig ob sie in dem für unabhängig erklärten Reservat, in einem der anderen Reservate oder aber im übrigen Südafrika leben, ihre südafrikanische Staatsangehörigkeit und erlangen diejenige des Reservates, dem sie ethnisch zugerechnet werden. Diese Politik hat Connie Mulder, einst mächtiger Kronprinz des seinerzeitigen Premierministers B. J. Vorster, so umschrieben ". . wenn unsere Politik logisch zu Ende geführt sein wird, dann wird es keinen schwarzen Menschen mehr mit südafrikanischer Staatsbürgerschaft geben $"{ }^{9}{ }^{9}$ Margaret Nash, eine sehr engagierte weiße Bürgerrechtlerin, äußerte von dieser Politik treffend, daß im 20. Jahrhundert das Gesetzbuch die Rolle spielt, die im 19. Jahrhundert Gewehr und Pferde gespielt haben: Der weiße Mann führt mit Hilfe seiner zu diesem Zweck geschaffene Gesetz Krieg gegen den schwarzen Mann. ${ }^{10}$ Keines der "unabhängigen" Reservate ist bislang von einem Drittstaat diplomatisch anerkannt worden. Derzeit werden in Südafrika Pläne verwirklicht, Südafrika und die "unabhängigen" Reservate zu einer Konföderation von Staaten des südlichen Afrikas zusammenzufassen. Wenn irgend möglich, so will Pretoria noch weitere Staaten in diese Konföderation hineinnehmen. Gedacht ist vor allem an Swaziland, aber auch an eine nicht von SWAPO regierte unabhängige Republik Namibia.

Für die gemischtrassigen ("Coloureds") und indienstämmigen ("Inder") Südafrikaner sah die Politik Pretorias ursprünglich vor, daß, parallel zu den Exekutiv- und Legislativorganen der Weißen, entsprechende Körperschaften für die beiden braunen Gruppen eingerichtet werden sollten." Hier sprach man von "paralleler Entwicklung", bei den Schwarzen hingegen von "getrennter Entwicklung". Auf die Finalität dieser Entwicklung angesprochen, äußerte C. Mulder, daß sich Parallelen niemals träfen. Die fehlende Bereitschaft der Coloureds wie auch der Inder, die ihnen von Pretoria zugedachten, substanzlosen Positionen zu bekleiden, ließ die Regierung dann nach neuen Wegen suchen, das "Selbstbestimmungsrecht" beider Gruppen zu verwirklichen.

9 Hansard (Kapstadt) Nr. 2, Spalte 579 (7. Februar 1978).

10 "Black Uprooting from 'Whiter SA", S. 41, hrsg. vom South African Council of Churches, Braamfontein, 1980.

11 Einzelheiten bei Klaus Frhr. von der Ropp "Republik Südafrika: Die politische Stellung der 'Coloureds' im System der Parallelen Entwicklung" in Int. Afrika Forum (München) 9-10/1972, S. 552-558; ders. "Republik Südafrika: Die politische Stellung der Inder` im System der Parallelen Entwicklung " in Int. Afrika Forum 11-12/1972, S. 660-666. 


\section{2) Zur Entstehungsgeschichte der neuen Verfassung}

Bereits die 1973 von Pretoria eingesetzte "Commission of Inquiry into Matters Relating to the Coloured Population Group", die sog. E. Theron Commission, empfahl, diese heute ca. 3 Mio (etwa 10 v. H. der Gesamtbevölkerung) starke Gruppe viel stärker in das soziale, wirtschaftliche und auch politische System der Weißen einzubringen. ${ }^{12}$ Die Regierung B. J. Vorsters bereitete diesen Vorschlägen, soweit sie den künftigen politischen Status der Coloureds betrafen, aus ideologischen Gründen ein drittklassiges Begräbnis. Die schwarze Rebellion von 1976/77, die die großen Sympathien vieler jugendlicher Coloureds für den Kampf der Schwarzen deutlich gemacht hatte, ließ die Regierung aber dann doch aktiv werden. Bereits Ende 1976 setzte sie ein Kabinetts-Komitee ein, das unter der Leitung des seinerzeitigen Verteidigungsministers P. W. Botha Úberlegungen zu "possible and desirable adjustments to the existing constitutional order . . . in respect of the political system for the Coloured and Indian communities" anstellten sollte. Entsprechend wurde im August $1976^{13}$ der Fraktion der regierenden NP ein Plan vorgelegt. Er wies zwei Charakteristika auf: Die Schaffung des Amtes eines nur indirekt gewählten, mit außerordentlich weitgehenden Kompetenzen ausgestatteten (weißen) Exekutivpräsidenten. Wahlberechtigt waren danach alle Weißen, Coloureds und Inder. Ubrigens wurde jetzt auch anderweitig eine deutliche Tendenz sichtbar, die staatliche Macht zu zentralisieren: der State Security Council (SSC), vormals nur einer von vier Kabinettsausschüssen, wurde zu einem Gremium ausgebaut, das sich mit Sicherheitspolitik im weitesten Sinn befaßte. Auf Einzelheiten wird weiter unten einzugehen sein.

Ferner sah der hier interessierende Plan die Einrichtung von drei voneinander unabhängigen Parlamenten vor, je eines für die Weißen, die Coloureds und die Inder. Deren angebliche Gleichberechtigung stand jedoch nur auf dem Papier. So verfügte beispielsweise nur das Parlament der Weißen über Budgethoheit. Jede der drei Bevölkerungsgruppen sollte nach diesen Vorstellungen ferner ihr eigenes Kabinett bekommen. Diese Legislativ- und Exekutivorgane waren nach den Prinzipien Teilautonomie und Gruppenrecht auf dem Papier für die "eigenen" Angelegenheiten der jeweiligen Gruppe zuständig. Da aber nur das Parlament der Weißen über eigene Einnahmen verfügte und über deren teilweise Weiterleitung an die beiden anderen Parlamente entschied, waren seine Mitglieder gleicher als die Mitglieder der anderen Parlamente. Bei den "allgemeinen" Angelegenheiten sollten die drei Parlamente, wenn irgend möglich, im Konsensus entscheiden. War dieser nicht zu erzielen, so sollte letztlich immer das Votum der Weißen den Ausschlag geben. Besondere Beachtung verdiente, daß infolge der Dreiteilung der Legislative es ausgeschlossen war, daß sich Parlamentarier der weißen Opposition mit den Mitgliedern der beiden braunen Parlamente verbündeten, um eine Entscheidung der weißen Mehrheitspartei zu Fall zu bringen. Die letztere würde, gemeinsam mit der zumindest über-

12 S. dazu S. T. van der Horst (Hrsg.) "The Theron Commission Report/A Summary", Johannesburg, $1976 ;$ O. D. Wollheim "Theron Commission: Comment", Johannesburg, 1977.

13 South African Institute of Race Relations (Hrsg.), "A Survey of Race Relations in South Africa 1976", Johannesburg, 1977, S. 7-10. 
wiegend weißen Exekutive, letztlich immer das alleinige Sagen haben. In späteren Jahren nannte die NP, in Abgrenzung ihrer eigenen Vorstellungen von jenen der PFP, die eine tatsächliche, institutionalisierte Teilung der staatlichen Macht unter den verschiedenen Bevölkerungsgruppen forderten ("Konkordanzdemokratische Ordnung"), ein System der "gesonde magsdeling", d. i. der "gesunden Machtteilung". Die Vorstellungen der PFP hingegen waren in den Augen der NP "ongesond ". Uberzeugend nannten Theo Hanf und Heribert Weiland vom Arnold-Bergstraesser-Institut/Freiburg das von der NP propagierte System ein solches der Schein-Konkordanz. ${ }^{14}$

Für die Schwarzen (ca. 70 v. H. der Bevölkerung), so die Empfehlungen, sollten alle wesentlichen Elemente der bisherigen Politik fortgeführt werden.

Die Vorstellungen des Kabinett-Komitees stießen auch bei kooperationsbereiten Coloureds, Indern und Schwarzen auf kompromißlose Ablehnung. Denn man sah, daß jetzt erstmals der Versuch unternommen wurde, die Politik der Apartheid gar verfassungsrechtlich zu verankern. Bereits damals war unter den Coloureds und den Indern die Furcht verbreitet, das weiße Südafrika wolle sich ihrer zu einem möglichst niedrigen Preis bedienen, um die in aller Augen unvermeidliche große Revolution der Schwarzen mit Aussicht auf Erfolg zertreten zu können. Zwar wurde 1979 noch das "Republic of South Africa Constitution Draft Billı veröffentlicht, jedoch nicht vom Parlament verabschiedet. Es wurde zur weiteren Beratung einer neugebildeten Kommission zugewiesen. Es hat allerdings den Anschein, als sei schon in jener Zeit die Entscheidung gefallen, unter Aufgabe der bisherigen parlamentarischen Ordnung (der Weißen) ein spezifisch südafrikanisches politisches System zu entwickeln. Geistreich sprach der Johannesburger Politikwissenschaftler Zolman Press davon, Südafrika bewege sich verfassungsrechtlich "von Westminster nach Southminster". Diese neue Kommission setzte sich, unter Führung von Innenminister A. Schlebusch, aus (ausnahmslos weißen) Parlamentariern der Regierungs- und Oppositionsparteien zusammen. Die Schlebusch-Kommission schlug dann schon im folgenden Jahr die Einsetzung eines "President's Council" (PC) vor, in welchem Weiße und kooperationsbereite Coloureds und Inder Empfehlungen für eine neue Verfassung erarbeiten würden. Der PC nahm seine Arbeiten auf, die Mehrzahl seiner braunen Mitglieder waren allerdings Quislinge.

Trotzdem erarbeitete der PC unter Federführung eines früheren NP-Parlamentariers, des sehr fähigen Denis Worrall, beachtenswerte Vorschläge. Die Arbeit des PC wurde allerdings ständig durch Interventionen des zuständigen Ministers, Chris Heunis, behindert. Der letztere schätzte die Atmosphäre in der NP zutreffender ein und versuchte daher, jeden wirklichen Reformansatz zu verhindern. Zunächst vermochte sich jedoch Worrall durchzusetzen, was anhand der im Mai 1982 veröffentlichten Empfehlungen auszumachen war. ${ }^{15}$ Ähnlich den Vorschlägen aus den Jahren 1977/79 sahen die des PC die Schaffung des Amtes eines mit sehr großer Machtfülle ausgestatteten Exekutivpräsi-

14 "Konkordanzdemokratie für Südafrika? Zur Bedeutung der neueren verfassungspolitischen Debatte" in Europa Archiv 23/1978, S. 755-770.

15 "First Report of the Constitutional Committee of the President's Council", Cape Town. 
denten vor. Man sprach allgemein von einer de Gaulle-Option. Des weiteren schlug der PC jedoch vor, daß, wenn auch nach getrennten Wahllisten zu ermittelnde, Volksvertretern der weißen und braunen Gruppen in ein und demselben Parlament und in ein und demselben Kabinett arbeiten und im Konsensusverfahren entscheiden sollten. Was die Schwarzen anbelangt, so kam der PC zu dem Ergebnis, daß es den Weißen und Braunen einerseits und den Schwarzen andererseits im Kulturellen, Sozialen, Politischen und Wirtschaftlichen einfach an jenem gemeinsamen Nenner fehle, ohne den selbst ein noch so dezentralisiertes gemeinsames Staatswesen nicht funktionsfähig sei. Für die Schwarzen empfahl der PC daher ein System der "multiplen ausgehandelten Teilung", d. h. die vertragliche Neufestlegung der Grenzen zwischen Südafrika einerseits und den Reservaten andererseits. In jener Zeit arbeitete übrigens eine vom Kapstädter Parlament eingesetzte Kommission (Hennie van der Walt-Kommission) an Uberlegungen zu einer geographischen und ökonomischen Konsolidierung der Reservate hin zu einigermaßen lebensfähigen Staaten. Dieser Studie erging es allerdings nicht anders als einer analogen Arbeit, die auf Initiative der damaligen Regierung Hendrik F. Verwoerds angefertigt worden war: aus Sorge vor einem weißen "backlash" wurde sie noch nicht einmal veröffentlicht, geschweige denn in die Tat umgesetzt.

Innerhalb und außerhalb Südafrikas, in der politischen wie wissenschaftlichen Diskussion, wurde nun abermals die Frage aufgeworfen, ob es in Südafrika tatsächlich an jenem gemeinsamen Nenner fehle. Immerhin kamen die kleine, jedoch kampferprobte PFP wie auch, was natürlich viel wichtiger war, eine von M. Gatsha Buthelezi berufene, mit hervorragenden in- und ausländischen Experten besetzte Kommission hier zu der Erkenntnis, daß dieser gemeinsame Nenner doch vorliege. ${ }^{16}$

Bahnbrechende Arbeit hat in diesem Bereich der Politikwissenschaften der in den USA lehrende Niederländer Arend Lijphart geleistet. ${ }^{17}$ Danach sind die folgenden Punkte mehr oder weniger Voraussetzung für die Funktionsfähigkeit einer Konkordanzdemokratie: die einzelnen Bevölkerungssegmente sind numerisch etwa gleich stark. Sie haben Erfahrungen im Zusammenleben gewonnen. Vom wirtschaftlichen Entwicklungsniveau her sind die einzelnen Bevölkerungsgruppen nicht durch einen allzu tiefen Graben getrennt. Dasselbe gilt für die Einkommens- und sonstigen Vermögensverhältnisse. Es existiert ein gemeinsamer äußerer Feind. Imgrunde bestreitet niemand, auch Lijphart selbst nicht, daß eigentlich all diese Voraussetzungen in Südafrika nicht erfüllt sind. So ist man versucht, den theoretischen Rahmen der südafrikanischen Realität anzupassen. Zwei führende Vertreter dieser Lehre, Van Zyl Slabbert und David Welsh, argumentieren hier nicht mehr akademisch sondern nur noch politisch "The only hope that some narrow basis for consensus could be established and consolidated is if the antaginists have a glimp-

16 S. "Report of the Constitutional Committee of the Progressive Federal Party . . "; "Buthelezi Commission Report", 1982, Durban.

17 Arend Lijphart "Majority Rule versus Democracy in deeply divided Societies" in Politikon (Pretoria) 2/1977, S. 113-126. 
se into the abyss of violence, economic disaster and all the miseries that go with unrestrained conflict $" 1{ }^{18}$

Nordirland, Zypern, das seinerzeitige Britisch-Indien und das frühere Mandatsgebiet Palästina haben deutlich gemacht, daß selbst konkordanzdemokratische staatliche Strukturen nicht jede Kluft zwischen Bevölkerungsgruppen zu überbrücken vermögen.

Die südafrikanische Gesellschaft, in der mit den weißen und schwarzen Afrikanern die Erste und die Vierte Welt aufeinandertreffen, ist gewiß sehr viel tiefer gespalten, als Außenstehende sich dies in der Regel vorzustellen vermögen. Die Kluft, die sich zwischen ihnen auftut, wurde in den mehr als drei Jahrzehnten der unangefochteten Herrschaft der NP gewiß nicht erst geschaffen, sie wurde jedoch durch deren Politik erheblich vertieft. Wie tief diese Kluft aber bereits vor 1948, dem Jahr der Machtübernahme durch die NP war, mag sich aus dem Umstand ergeben, daß schon Mitte der 30er Jahre ein hochausgewiesener weißer Liberaler die Frage aufwarf, ob nicht einer "gerechten" Teilung des Landes (später sprach man hier vom Modell Indien/Pakistan) der Ausweg aus einem sonst unlösbaren Dilemma liegen könne. ${ }^{19}$

Hier müssen einige wenige Bemerkungen genügen, um die Polarisierung der südafrikanischen Gesellschaft aufzuzeigen. Es fehlt im Grunde an jedem sozialen Kontakt zwischen Schwarz und Weiß, an jeder Kommunikation. Eine Seite ist kaum mit den Problemen der anderen vertraut und will dies auch gar nicht sein. Von der Wiege bis zur Bahre leben die Menschen nicht mit- sondern nebeneinander. Diese Trennwände sind noch viel höher als beispielsweise die Trennwände, die in der Bundesrepublik Deutsche und Türken trennen: getrennte Wohngebiete, getrennte Schulen aller Stufen, getrennte Restaurants, getrennte Strände, getrennte Verkehrsmittel, getrennte Hospitäler, getrennte Friedhöfe usw.

Immer wieder, und selbst heute noch, wurden von schwarzen und weißen Liberalen Versuche unternommen, einen Dialog über die ethnischen Grenzen hinweg zu führen. Dazu zwei Beispiele: Der frühere Chefredakteur der liberalen Johannesburger Rand Daily Mail (RDM), A. Sparks, sah in seiner Tageszeitung stets "eine Brücke über die Grenzen der rassischen Ghettos hinweg “. Der RDM ist zu danken, daß einer größeren Offentlichkeit Einzelheiten über von Armeeangehörigen in SWA/Namibia begangene Folterungen; über die gewaltsame Abschiebung ("relocation") von Schwarzen in die Reservate; über die Aussichtslosigkeit der Deportierten, einen noch so bescheidenen Lebensunterhalt zu finden; über Akte der Willkür und Brutalität der Polizei gegen die Bewohner illegal im "weißen" Südafrika errichteter Lager (K. T. C., Crossroads, Nyanga usw.); über die Entwicklung schwarzer Gewerkschaften; über die Behinderung von deren Arbeit und selbst über Aktivitäten bewaffneter Widerstandsgruppen usw. bekannt wurden. Aber Sparks gelang dennoch ein Brückenschlag nicht. Letztlich scheiterte er daran, daß der schwarze Rebell, etwa der ANC-Aktivist, für die Masse der Schwarzen eine Bote der

18 Frederik Van Zyl Slabbert and David Welsh "South Africa's Options/Strategies for Sharing Power", S. 119, Cape Town, 1979.

19 S. R. F. (Alfred Hoernlé "Anatomy of Segregation" in Race Relations (Johannesburg) 1/1936, S. 14-21. 
Freiheit, für die weitaus meisten Weißen aber ein Terrorist ist. Sehr viele Schwarze erhoffen vom ANC Befreiung. Viele der wenigen extrem kritischen Weißen sind jedoch nicht frei von Furcht vor der Herrschaft der schwarzen Revolutionäre. So zwangen die Interessen ihrer Leser die RDM schon vor Jahren, getrennte Ausgaben für die Schwarzen (RDM extra) und die Weißen (RDM) herauszugeben. Insbesondere in der katholischen Kirche wurden unter Leitung eines bedeutenden Liberalen, des Erzbischofs von Durban und Vorsitzenden der Southern African Bishops' Conference, Denis Hurley, entschiedene Versuche unternommen, schwarze und weiße Christen zusammenzuführen. Allem Anschein nach stoßen auch diese Versuche auf alle Arten von großen Schwierigkeiten. So wurde etwa vor einiger Zeit bekannt, daß es selbst in Klöstern immer wieder zu unüberwindbaren Spannungen zwischen weißen und schwarzen Ordensschwestern kommt. Nach alledem kann es nicht Wunder nehmen, daß es, außer Repräsentanten englischsprachiger, "integrierter" Kirchen, auch schwarze Katholiken waren, die Anfang 1980 in Hammanskraal/Pretoria erstmals über die Gründung einer (überkonfessionellen!) Bekennenden Kirche sprachen, einer Kirche des Protestes, des Widerstandes, der Befreiung.

Vor diesem Hintergrund sind übrigens auch die Aufrufe vieler weißer und schwarzer Kirchenführer zu sehen, bei dem Verfassungs-Referendum vom 2. November 1983 mit Nein zu stimmen, da die neue Verfassung die Apartheid festschreibe. Sehr viele Wahlberechtigte mißachteten nämlich diese Aufrufe und gaben $\mathrm{P}$. W. Botha ihre Stimme.

Bereits lange vor der Veröffentlichung der Empfehlungen des PC waren diese in der NP sehr umstritten. Es erschien möglich, daß die Partei sie mehrheitlich als zu "links" ablehnen werde. Unmittelbarer Anlaß für den dann im März 1982 vollzogenen Bruch innerhalb der NP war die Veröffentlichung eines Artikels von Jan Groblar (NP) in einer Propagandabroschüre seiner Partei. ${ }^{20}$ Der damalige Vorsitzende der NP in Transvaal, Andries Treurnicht, verließ daraufhin zusammen mit 15 weiteren Parlamentariern die NP. Allem Anschein nach bestand jetzt für die NP die Gefahr, daß Treurnicht jetzt sehr viele weitere Abgeordnete und sonstige Parteimitglieder sowie die Mehrheit der NPWähler politisch an sich binden würde. Dies umso mehr, als Treurnicht kurze Zeit später mit seinen Gefolgsleuten die latent sehr starke Konserwatieve Party (KP) gründete. Sie rekrutiert ihren Anhang überwiegend aus dem Afrikanerdom. Politisch einig mit ihr wissen sich jedoch sehr viele der allerdings nicht wahlberechtigten über 500000 Portugiesen, die aus Angola und Mozambique nach Südafrika geflohen sind. Dasselbe gilt für die bald 100000 "exRhodesians", weilße Bürger Zimbabwes, die nach dem Machtwechsel von April 1980 zwar nicht vertrieben wurden, aber doch für sich und ihre Kinder in Zimbabwe Robert G. Mugabes keine Zukunft sahen und daher nach Südafrika auswanderten. Es ist damit zu rechnen, daß das Gros beider Gruppen über kurz oder lang die südafrikanische Staatsangehörigkeit und damit auch die Wahlberechtigung erwerben wird. Die exRhodesians haben übrigens bereits heute einen starken Einfluß auf das Denken 
der aber ohnehin nur nach einem verbreiteten Fehlurteil "liberalen" englischsprachigen weißen Südafrikaner. "Boer" und "Brit" eint heute viel mehr als sie trennt.

Spätestens die Gründung der KP ließ P. W. Botha vor Treurnicht zurückweichen. So ließ er für den Bundeskongreß der NP in Bloemfontein (Mitte 1982) Richtlinien für eine neue Verfassung erarbeiten. Deren Kern war der Verzicht auf nahezu alle von Worralls Vorschlägen und eine Rückbesinnung auf die eigenen Úberlegungen aus den Jahren 1977/79. Denis Worrall wurde seines Amtes enthoben und zum Botschafter in Australien ernannt. An seine Stelle trat der farblose und fügsame Schalk van der Merwe. Unter seiner Führung erarbeitete der PC einen im November 1982 veröffentlichten "Zweiten Bericht". Dieser lag ganz auf der Linie dessen, was die NP zu verkraften im Stande war. ${ }^{21}$ Sie deckten sich nämlich sehr weitgehend mit den Vorstellungen von 1977/79. 1983 wurden sie im Parlament debattiert und, gegen das Votum von PFP und KP, mit zwei Drittel der Stimmen angenommen.

Anfang November 1983 stimmten bei einem Referendum zwei Drittel der wahlberechtigten Weißen (nur sie wurden befragt!) bei einer Wahlbeteiligung von ca. $75 \mathrm{v}$. H. dieser neuen Verfassung zu. In Südafrika selbst wie auch im Ausland wurde der Ausgang des Referendums oft dahingehend interpretiert, daß er die relative Schwäche von KP und HNP ("die Schwäche des 'konservativen Lagers") gezeigt habe. Eine Bewertung, die vor dem Hintergrund des Zurückweichens der NP vor der KP/HNP schlicht unsinnig erschient.

\section{3) Die Strukturen der neuen Verfassung}

Das weiße Südafrika, insbesondere sein afrikanser Kern, sah und sieht sich noch stets als ein zutiefst christlich geprägtes Volk. So heißt es auch in der Präambel der neuen Verfassung 22 "IN HUMBLE SUBMISSION to Almighty God . . . we are conscious of our responsibility towards God and man ... goals: To uphold Christian and civilized standards . . . To respect, to further and to protect the self-determination of population groups and peoples ...". Wohl das gesamte schwarze Südafrika sieht darin ein Bekenntnis zur Apartheid. Und verbreitet ist unter schwarzen Christen die Uberzeugung, daß Apartheid Ketzerei, ja Sünde sei.

Das südafrikanische Parlament wird in Zukunft drei Kammern haben, das House of Assembly der Weißen (178 Mitglieder), das House of Representatives der Coloureds (85) und das House of Deputies (45) der Inder. Das hier niedergelegte Zahlenverhältnis von 4 zu 2 zu 1 gilt auch für andere Institutionen, etwa das Kabinett. Jede der Kammern ist für die (in Anhang 1) zu der Verfassung aufgezählten) "eigenen" Angelegenheiten allein zu-

21 "Second Report of the Constitutional Committee of the President's Council", Cap Town.

22 Zu der neuen Verfassung s. aus dem deutschsprachigen Schrifttum vor allem Robert von Lucius "Von der Alleinherrschaft zur Vorherrschaft " in FAZ vom 31. Oktober 1983, S. 12. Aus Südafrika s. vor allem Nic J. J. Olivier "The Constitutional Proposals of the South African Government", von der FNS/Bonn Mitte 1984 zu veröffentlichenden Manuskript; Andre du Toit "The case of 'reform equals one-party rule" and "A scheme for white domination" in RDM (Johannesburg) vom 2. bzw. 3. Dezember 1982. 
ständig. Aus Rücksicht auf die KP, die HNP und die vielen Reaktionäre in den eigenen Reilhen ist die Liste der eigenen Angelegenheiten sehr umfangreich. Sie umfaßt zum Beispiel das Erziehungswesen aller Stufen und selbst die Landwirtschaft: wenn Parallelen sich schon treffen, dann eben möglichst selten. Anderenfalls wäre ein Zustand der "ongesonde magsdeling" erreicht. Die drei Kammern haben, im Konsensusverfahren, gemeinsam über die "allgemeinen" Angelegenheiten (Außen-, Sicherheits- und "Schwarzen"Politik usw.) zu entscheiden. Verhängnisvoll erscheint, daß der (in der Praxis, wenn auch nicht kraft Gesetzes) stets weiße Staatspräsident im Streitfall darüber zu entscheiden hat, ob es sich bei einer politischen Frage um eine eigene oder eine allgemeine Angelegenheit handelt. Seine Entscheidung ist gerichtlich nicht nachprüfbar.

Von großer Bedeutung werden in Zukunft die "Gemeinsamen Ausschüsse" der drei Kammern sein, die für die Erarbeitung gemeinsamer Positionen zu den allgemeinen Angelegenheiten eingerichtet werden. Ihnen werden möglicherweise auch Mitglieder der Oppositionsparteien angehören. Möglich ist, daß die Geschäftsordnungen dieser Ausschüsse vorsehen werden, daß deren Debatten nicht öffentlich und damit der Berichterstattung durch die Presse weitgehend entzogen sein werden. Southminister unterscheidet sich nicht zuletzt durch seine ausgeprägt autoritären Züge von Westminster.

Können die Gemeinsamen Ausschüsse einen Konsensus nicht erreichen, so greift der "Präsidialrat" ein, wohl nur aus optischen Gründen nicht der Staatspräsident. In der Sache wird das allerdings kaum einen Unterschied machen. Denn in jedem Fall hätte die weilße Mehrheitspartei, entgegen dem elementarsten Prinzip einer jeden konkordanzdemokratischen Ordnung, hier das letzte Wort. Denn der Staatspräsident wird faktisch von den 50 weißen Mitgliedern des "Wahlkollegiums" gewählt. Die letzteren werden alle von der Mehrheitspartei im House of Assembly benannt. Und im Präsidialrat wird der Staatspräsident durch die Auswahl der von ihm zu ernennenden 25 (von 60) Mitgliedern zu verhindern wissen, daß es hier zu ongesonden Machtkonstellationen kommen wird. Wahrscheinlich werden die Weilen allerdings, trotz aller gebotenen Rücksichtnahmen auf KP, HNP usw. ihre rechtlichen Befugnisse nicht voll ausspielen. Denn anderenfalls würde die allem Anschein nach nur kleine Zahl kooperationsbereiter Coloureds und Inder sicher noch weiter schrumpfen. In der Notwendigkeit, sie für eine aktive Mitarbeit zu gewinnen, liegt für die Coloureds und die Inder die Chance, daß ihre Interessen bei der bislang noch nicht geregelten Art der Finanzzuteilung, der Grundlage jeder politischen Aktivität, nicht übergangen werden.

Die neuen Exekutivorgane sind mehr oder weniger das Spiegelbild der neuen Legislative. Dem Kabinett werden, im Verhältnis 4 zu 2 zu 1, Weiße, Coloureds und Inder angehören. Sie sind für die allgemeinen Angelegenheiten zuständig. Die Kabinettsmitglieder jeder der drei Bevölkerungsgruppen, nur sie, bilden die (drei) Ministerräte, die jeweils für eigene Angelegenheiten zuständig sind. Das Kabinett ist nicht dem Parlament sondern dem Staatspräsidenten verantwortlich. Der letztere wird trotz seiner übergroßen Machtfülle, anders als der Präsident der französischen Republik und faktisch auch der der USA, nicht unmittelbar von der Bevölkerung sondern dem bereits erwähnten Wahlkollegium gewählt. Autoritäre Herrschaftsstrukturen werden ferner bei der im letzten Jahr- 
zehnt immer bedeutsamer gewordenen Position des State Security Council (SSC) erkennbar. ${ }^{23}$ Offiziell nur eines von mehreren Kabinettskomitees, gehören ihm, in Zukunft unter dem Vorsitz des Staatspräsidenten, die (weißen/braunen) Spitzen einiger Schlüsselministerien und deren höchste Beamte an. Im Schrifttum wurde in jüngerer Zeit davon gesprochen, daß hier alle Befehlsstränge zusammenliefen, daß hier, also nicht im Kabinett, auch die Entscheidungen für die Bekämpfung des vom "internationalen Kommunismus" gegen Südafrika getragenen "total onslaught" gefällt würden. Der Amerikaner Kenneth W. Grundy verglich die Befugnisse des SSC mit denen des Politbüros in einem kommunistischen Staat. Es bleibt abzuwarten, ob unter diesen Umständen Coloureds und Inder, welche Positionen auch immer sie einnehmen werden, tatsächlich die Politik mitgestalten werden. Sheena Duncan, streitbare Vorsitzende der sehr engagierten Bürgerrechtsbewegung Black Sash, urteilt wohl zutreffend über Southminster, wenn sie schreibt "Power is being centralised not shared ". ${ }^{24}$

\section{4) Zu den Reaktionen der Schwarzen und Braunen}

In "verligten" Kreisen der NP wird häufig die Hoffnung ausgedrückt, daß P. W. Botha nach dem für die NP erfolgreichen Ausgang des Referendums eine "hidden agenda" der

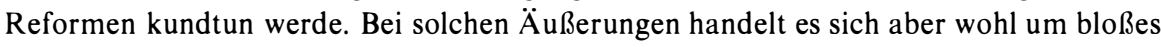
Wunschdenken: Für die Schwarzen wird es bei der Politik der Getrennten Entwicklung verbleiben. Deren Komponente "große Apartheid" gilt unverändert. Bei der kleinen Apartheid wurden, zugunsten der sog. section-tenners, d. s. die Schwarzen mit einem unbegrenzten Aufenthaltsrecht im "weißen" Südafrika, allerdings seit Ende der 70er Jahre eine Reihe wichtiger Neuerungen eingeführt: Abschaffung der gesetzlich verankerten "job reservation", bessere Möglichkeiten der schulischen und beruflichen Ausbildung, bessere Wohnverhältnisse usw. Die anderen Schwarzen, es handelt sich um viele Hunderttausende, werden nach Ablauf ihrer zeitlich begrenzten Aufenthaltsgenehmigung jetzt verstärkt in die Reservate abgeschoben. Das gilt auch für solche Schwarzen, die, oft als Eigentümer des von ihnen bebauten Grund und Bodens ("black spots" im weißen Südafrika) lebten, dann aus ideologischen Erwägungen enteignet und abgeschoben wurden. Hier herrscht nach der "Umsiedlung" von etwa 3,5 Mio Menschen unvorstellbares Elend. ${ }^{25}$ Nach Schätzungen kirchlicher und anderer Stellen werden dennoch in den kommenden Jahren weitere 2 Mio Menschen zwangsweise umgesiedelt werden. Viel bedeutsamer als die genannten Modifizierungen ist die durch den sog. Wiehahn-Bericht einge-

23 S. dazu Deon Geldenhuys and Hennie Kotzé, "Aspects of Political Decision-Making in South Africa" in Politikon (Pretoria), Juni 1983, S. 33-45; Kenneth W. Grundy, "The Rise of the South African Security Establishment. An Essay on the Changing Locus of State Power", hrsg. vom SAIIA, Braamfontein/SA 1983. Im Anschluß an diese Arbeiten s. Winrich Kühne, "Südafrika in den 80er Jahren: Militarisierung einer Gesellschaft und einer Region", SWP/Ebenhausen, 1984.

24 "Reform: Quo Vadis?" in South Africa International (Johannesburg) 10/1982, S. 99-111 (100).

$25 \mathrm{Vgl}$. dazu die sehr engagierte Berichterstattung in Sash (Johannesburg), South African Outlook (Rondebosch), Ecunews (Johannesburg), Diakonia (Durban) sowie die Jahresberichte der regionalen Beratungsbüros von Black Sash. 
leitete Reform des schwarzen Gewerkschaftslebens. Es umfaßt alle schwarzen Arbeitnehmer, also außer den section-tenners auch die Wanderarbeiter und die Pendler.

Die neu entstandenen Gewerkschaften, so uneinig sie heute auch (noch?) sein mögen, sie gaben ihrer kompromißlosen Zurückweisung von Southminster unübersehbar und unüberhörbar Ausdruck. Auf der selben Linie lagen die Äußerungen im Grunde aller schwarzen Kirchenführer und Politiker. Hier unterscheidet sich die Sprache des erbärmlichsten Quislings kaum noch von der des Aktivisten einer der militanten Befreiungsbewegungen. ${ }^{26}$ Durch den weitgehenden Boykott der Wahlen zu den mit neuen Befugnissen ausgestatteten kommunalen Selbstverwaltungskörperschaften haben die hier wahlberechtigten Schwarzen, d. s. die section-tenners, Anfang Dezember 1983 ein Kernstück der neuen Ordnung, den Black Local Authorities Act, zurückgewiesen. So gingen in Soweto, der wichtigsten schwarzen Stadt im "weißen "Südafrika nur 11 v. H. der Wahlberechtigten zu den Urnen. Häufig lehnten übrigens auch die Wortführer der Coloureds ${ }^{27}$ und der Inder Southminister in einer sehr harten Sprache ab. Die große Mehrheit beider Gruppen fürchtet sich wohl immer noch vor zumindest einer uneingeschränkten Herrschaft der schwarzen Revolutionäre. Sie ist jedoch nicht bereit, den Weißen bei der Aufrechterhaltung eines auf Dauer unhaltbaren Systems behilflich zu sein. Das ist auch in Pretoria und bei den kooperationswilligen Coloureds und Indern bekannt. Folglich wird es ein Referendum für die Coloureds und die Inder kaum geben. Anderenfalls käme es wohl zu einem Debakel für die Regierung.

\section{Schlußbemerkungen}

Es bedarf keiner prophetischen Gaben, um Southminster auf die Dauer keine Erfolgschancen einzuräumen. Angesichts der Unfähigkeit der Südafrikaner, ihre Probleme selbst durch Verhandlungen beizulegen, wie auch der Unfähigkeit des Westens, hier zu vermitteln, besteht die Versuchung, den Ausweg aus dem südafrikanischen Dilemma in, wie es Gustav Adolf Sonnenhol ${ }^{28}$ einmal ausgedrückt hat, einer post-katastrophalen Lösung zu suchen, nämlich der Teilung des Landes in einen schwarzen und einen weiß/ (braunen) Staat. Darüber liegt heute bereits viel Schrifttum vor. ${ }^{29} \mathrm{Da}$ auch in Pretoria

$26 \mathrm{Zu}$ der Stärke der einzelnen Gruppen s. die in Anm. 1) genannte Studie, die allerdings gerade in dem hier interessierenden Punkt veraltet sein wird. S. weiter Tom Lodge "Black politics in South Africa since 1945", Johanresburg, 1983 sowie James Selfe "The State of Black Politics in South Africa Today", von der FNS/Bonn Mitte 1984 zu veröffentlichendes Manuskript.

27 S. etwa Allan Boesak "New deal seeks to entrench evil system" in Sash 5/1983, S. 7-9, 27.

28 In FAZ vom 4. Mai 1983 "Fünf Minuten vor Zwölf können lange dauern“. S. weiter F. Van Zyl Slabbert and David Welsh, a.a.O., S. 169.

29 Im Anschluß an den Beitrag des Amerikaners Edward A. Tiryakian "Sociological Realism: Partition for South Africa" in Social Forces, 1967. Dezember 208-221 siehe Jürgen Blenck und Klaus Frhr. von der Ropp "Republic of South Africa: Is Partition a Solution?" in SA Journal of African Affairs (Pretoria) 1/1977, S. 21-32; Gavin Maasdorp "Froms of Partition, S. 107-146 in Robert I. Rotberg and John Barratt (Hrsg.) in "Conflict and Compromise in South Africa", Lexington u. a., 1980; Paul L. Moorcraft "Towards the Garri- 
diese Möglichkeit als letzte Rückfallposition durchdacht wird, kann u. a. daráus gefolgert werden, daß die Regierung im West-Kap noch stärker als im übrigen "weißen" Südafrika bemüht ist, die Zahl der dort lebenden Schwarzen drastisch abzubauen. John Kane-Berman, ein sehr scharfer Kritiker seines Landes und heute Direktor des South African Institute of Race Relations, schrieb einmal treffend: ". . there no doubt exists at the back of the collective Nationalist mind the fear that one day the white man may have to trek back whence he came and the last thing he'd want to find is that the final bunker is full of blacks". ${ }^{30}$

son State" in F. M. Clifford Vaughan (Hrsg.) International Pressure and Political Change in South Africa, Cape Town, 1978, S. 86-105; Klaus Frhr. von der Ropp "Power Sharing versus Partition in South Africa" in Australian Outlook (Canberra) 2/1981, S. 158-168; ders. "Il Sudafrica dopo Zimbabwe" in Présence Libérale (Rom/Brüssel) 2/1981, S. 96-104. Vgl. auch Wolfgang H. Thomas "Südafrika zwischen Teilung und Integration" in Außenpolitik 3/1979, S. 301-322.

30 "The treatment of Nyanga's squatters revealed apartheid's final solution" in Race Relations News (Johannesburg) 1981. Oktober, S. 4; s. in diesem Zusammenhang "Voorgestelde gebiede vir blanke groeipunte" in Oranje (Nuusbrief von die Vereniging van Oranjewerkers) 1982. März, S. 1-3. Beachte dazu die auf S. 30 der in Anm. 29) genannten Studie (SA Journal of African Affairs) abgedruckten Landkarten. 


\title{
ABSTRACTS
}

\author{
The Republic of South Africa's New Constitution - from "Westminster" to "Southmin- \\ ster"
}

By Klaus Frhr. von der Ropp

The long-time watcher of the Southern African crisis scenarios in 1984 will come to the conclusion that white South Africa has regained its traditional position of power and self-confidence which it had lost after the collapse of the Portuguese empire in Africa, the coming to power of Robert G. Mugabe in Zimbabwe and the 1976/77 rebellion of black South Africa's youth. Foremost among numerous other factors, the failure of the West's Namibia and South Africa policy in 1977-81 contributed to this resurgence of strength. At the time the five members of the Western Contact group asked for a system of majority rule to be introduced in the former mandate territory of South West Africa/ Namibia. Three members of the group, namely the U.S., West Germany and Canada, held the view that this solution, intended for Namibia, would also serve as an example for the Republic of South Africa. Thus Western powers created high expectations among black South Africans and provoked fear even among the most liberal whites. As a consequence the existing polarisation between the white African nation and black South Africa was exacerbated. To most observers inside and outside South Africa it was hardly conceivable that the many progressive speeches of those years would not be followed by any action.

Today's debate on South Africa's new constitutional dispensation has to be seen against the above-mentioned background. Pretoria no longer sees the necessity of genuine political change. So, after long and intensive debates, a system of sham-consociationalism is established for white, 'coloured a and Indian South Africans, whereas the existing system of separation is maintained for the country's black majority. The fear therefore persists that, to quote Gatsha Buthelezi, future constitutional developments in South Africa are going to be a product of bullets and power.

\section{Oil, Economic Crisis and the State in Mexico}

\section{By Herbert Baumgartner}

Despite favourable conditions the Mexican economy recently tumbled into its most serious crisis since the 1930ies. Between 1978 and 1981 the oil industry was the key for high growth rates. PEMEX, the national oil company, experienced dynamic expansion, multiplying sales. Nevertheless it maintained inefficient structures and corruption became rampant. 